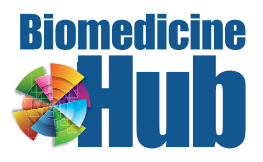

\title{
eHealth as a Facilitator of Precision Medicine in Epilepsy
}

\author{
Gianpiero L. Cavalleri ${ }^{a, c}$ Slave Petrovski ${ }^{d}$ Mary Fitzsimons ${ }^{a-c}$ \\ Norman Delanty ${ }^{a-c}$ \\ a Department of Molecular and Cellular Therapeutics, The Royal College of Surgeons in \\ Ireland, ${ }^{b}$ Department of Neurology, Beaumont Hospital, and ${ }^{\mathrm{C}}$ The FutureNeuro Research \\ Centre, Dublin, Ireland; ${ }^{\mathrm{d}}$ Department of Medicine, The University of Melbourne, Austin \\ Health and Royal Melbourne Hospital, Melbourne, VIC, Australia
}

\section{Keywords}

eHealth · Epilepsy · Precision therapeutics

\begin{abstract}
Epilepsy is a chronic neurological condition that affects approximately 50 million people worldwide. Current treatments are inadequate and around a third of patients continue to experience uncontrolled seizures. The genetic architecture of many of the epilepsies makes them amenable to next-generation sequencing technologies, enabling a molecular diagnosis in an increasing proportion of patients. As a result, rare but remarkable examples of precision therapeutics in epilepsy are emerging. Coordinated research efforts are required to increase the diagnostic yield of sequencing and translate diagnosis to improved prognosis. This review explores the potential of eHealth technologies in facilitating and accelerating precision therapeutics in epilepsy. We describe the state of the art in precision diagnostics and therapeutics in epilepsy and identify opportunities for eHealth to accelerate the realisation of precision therapeutics via patient registries, research-enabled electronic health records, and connected health solutions.

(c) 2017 The Author(s)

Published by S. Karger AG, Basel
\end{abstract}

\section{Introduction}

By placing the individual at the centre of clinical activity, health care professionals have long strived for precision medicine, preventing and treating human disease in a manner that takes individual variability in to account. However, the ability to truly characterise individual variability at both a genetic and environmental level is only now becoming 
possible via step-changes in DNA sequencing capacity and rapid developments in networked technology. This review outlines the potential for precision medicine in epilepsy, exploring features of the condition and associated research infrastructure that make it amenable to the clinical translation of genomics and expansion of precision therapeutics to the care pathway.

Up to one billion people worldwide suffer from neurological diseases. In Europe, neurological conditions represent a third of the total burden of all disease [1]. Much of this burden comes from ineffective and poorly informed treatment protocols, so-called "controlled trial and error." Up to now, the application of precision therapeutics in neurological disease has proven extremely challenging, as often the specific aetiology of a patient is simply unknown, leaving relatively little patient-specific data to guide clinical decision making. However, the application of next-generation sequencing across a diverse range of neurological diseases is shedding light on the underlying genetic architecture of specific neurological diseases, and in some cases, pointing to clear underlying pathogenic mutations in particular patients - so-called precision diagnostics. However, diagnostics is only part of the equation; the challenge is to identify an effective therapy based on that molecular diagnosis.

\section{What Is Required to Deliver Precision Therapeutics?}

Discovery in precision therapeutics requires excellent basic science connected to specialised clinical care. The role of basic science here includes improving the diagnostic yield of molecular tests and developing new, or matching existing therapeutics to a specific molecular diagnosis. Clinical integration of precision therapeutics requires that researchinformed specialist clinicians have access to appropriate diagnostic tests. Both discovery and clinical integration of precision therapeutics can be facilitated by eHealth technology. At a clinical level, eHealth infrastructure can provide for the collection and analysis of patient data to monitor treatment response. At the research level, eHealth infrastructure can provide rapid access to, and sharing of, high-quality patient phenotypes, critical for many research questions including discovery of clinically relevant genetic variation. Mobile technology, when linked to electronic patient records, offers the potential to enrich the phenotype further.

\section{Epilepsy as a Model for Precision Therapeutics in Neurological Disease}

Within neurological diseases, epilepsy can make a compelling case as a model disease for the development and expansion of precision therapeutics. First, the condition would benefit greatly from a precision therapeutics approach, particularly in the approximately one third of patients with refractory epilepsy [2]. Second, developments in sequencing technology allow a significant and growing proportion of cases to achieve a genetic diagnosis [3]. Third, the genetic architecture of the condition is complex, with mutations in any of over 30 genes sufficient to cause seizures [3]. Fourth, informative phenotypes exist across multiple experimental models to inform on the physiology of a particular molecular diagnosis [4], and fifth, the nature of the condition makes it amenable to connected health/ eHealth solutions [5]. There are already remarkable examples of the application of precision therapeutics in epilepsy, and consolidating critical infrastructure would accelerate discovery and expansion. 


\section{How We Traditionally Treat Epilepsy: Controlled Trial and Error}

There are currently over 20 anti-epileptic drugs available for the treatment of epilepsy, with two new drugs approved over the last 4 years (perampanel and brivaracetam). Currently there is much interest in the use of a cannabis extract, cannibidiol, as a further novel agent that is likely to gain regulatory approval in the near future. These drugs act by suppressing the most obvious manifestation of the epilepsies, seizures, and have been developed primarily via the Anti-Epileptic Drug Development program (www.pharmacy.utah.edu/add) sponsored by the United States National Institute of Neurological Disorders (NINDS), now based at the University of Utah under the directorship of Dr. Karen Wilcox.

The goals of treating individuals with epilepsy are: (a) maximise quality of life, (b) achieve seizure freedom, (c) avoid or minimise acute and chronic adverse side effects of anti-epileptic drugs, and (d) treat associated comorbidities. These goals are not always attainable. For example, one third of individuals with epilepsy will continue to experience seizures with a significant impact on quality of life [2]. Patients react to the various anti-epileptic drugs differently and many of the current therapeutics of epilepsy can be described as "controlled trial and error." Currently, no pharmacogenomic test exists that can aid the clinician in predicting optimum drug efficacy in an individual patient. Furthermore, only two pharmacogenomics tests (both based on HLA genotyping) are available to help predict serious adverse side effects of therapy, both related to carbamazepine hypersensitivity [6, 7]. In summary, the diagnostic tools and treatment options available to the epileptologist are insufficient to effectively control epilepsy in over a third of cases, and many of those individuals who achieve seizure freedom often experience side effects to the treatment.

\section{Next-Generation Sequencing in Epilepsy: Accurate Diagnosis as a Platform for Precision Therapeutics}

In contrast to the limited diagnostic value available from large-scale common variant genome-wide association studies [8], next-generation sequencing technologies have achieved high rates of molecular diagnoses for individuals living with epilepsy [9]. Among the rare paediatric epilepsies, for example, more than $30 \%$ of individuals with an epileptic encephalopathy obtain a molecular diagnosis on the basis of a single dominant-acting de novo mutation in a known epilepsy gene [10]. More recently, large samples of individuals with more common genetic generalised and non-acquired focal epilepsies were sequenced and published as part of an Epi4K international collaboration [11]. The Epi4K study highlighted ultra-rare epilepsy gene variants as carrying a significant epilepsy risk contribution in familial common epilepsies; up to $8 \%$ of familial non-acquired focal epilepsy cases showed a significantly elevated rate, over the control population, for ultra-rare variants across just five epilepsy genes: $D E P D C 5$, LGI1, PCDH19, SCN1A, and GRIN2A [11]. Many of the genes identified as important among common epilepsies overlap with known epileptic encephalopathy genes, confirming a shared genetic overlap between common and rarer severe epilepsies.

\section{Challenges with Interpretation of Sequence Data: Distinguishing Attractive from Definite}

However, greater accessibility to these sequencing technologies requires stricter standards for assigning pathogenicity. With the ready access of gene panel or exome data on patients, it is easy to leap to incorrect conclusions about an "attractive" variant in a known 
disease gene [12]. Thus, despite important advances made in identifying genes that contribute to epilepsy risk, interpreting the variants responsible for disease in an individual's genome remains challenging.

Robust variant classification guidelines like those proposed by The American College of Medical Genetics and Genomics (ACMG) have improved variant classifications by systematising the interpretation process [13]. As a result, occasionally an epilepsy gene variant classification is definitive. However, the majority class of variants reported in diagnostic sequencing remain "variants of uncertain significance" (VOUS). Despite being appropriately classified on the basis of current knowledge, once a VOUS is shared, the patient's clinician, patient, and family can generate their own impressions over the relevance of uncertain findings. This can be further complicated as epilepsy moves toward the use of genetically targeted therapies, and it becomes increasingly critical that the correct variant(s) contributing to disease risk in individual patients are identified [14]. This is best illustrated by the field of cancer genetics, which has shown that only through a robust approach to precision diagnostics has precision medicine become appropriately judged and subsequently successfully adopted in routine clinical management.

Next-generation sequencing technologies are now available to clinical labs, although there are disparities in accessibility of testing across health care systems. The resulting influx of patient genomic data has allowed for a more sophisticated understanding of the genetic architecture underlying epilepsy with and without an encephalopathy, and a deeper appreciation of the allelic series in individual epilepsy genes [15]. The results have also taught us about the expanding phenotypic spectrum associated with individual epilepsy genes and the diverse mechanisms by which epilepsy risk alleles can arise, including a major contribution to the epilepsies from germline and somatic de novo mutation events [16] and a higher parental-mosaic transmission contribution than had been previously expected [17].

\section{The Emergence of Precision Therapeutics in Rare Epilepsy Syndromes}

Although obtaining an accurate molecular diagnosis has important personal utility for many individuals and families living with epilepsy, with current knowledge a fraction of positive genetic diagnoses can also have a transformative effect on clinical management. There is a growing list of neurological disorders that already benefit from genetically targeted therapies, highlighting that some patients who obtain a correct molecular diagnosis might already be candidates for clinically relevant changes to management. One such early precision medicine example in epilepsy was the SLC2A1 association with early-onset "GLUT1 deficiency" absence epilepsy, a rare seizure disorder with a prevalence of around 1:83,000 people [18]. Patients with SLC2A1 epilepsy carry disruptions in the SLC2A1 gene that result in reduced glucose transportation through the blood-brain barrier, a condition called GLUT1 deficiency syndrome. Patients with this GLUT1 deficiency now use a ketogenic diet as a therapeutic intervention, resulting in the immediate cessation of seizures in many cases. The treatment of GLUT1 deficiency syndrome also represents an example of the potential for nonpharmacological precision therapy in epilepsy.

In this new and evolving era of genomic medicine, recent and ongoing international collaborative efforts have paved the way forward towards precision therapeutics [3]. The epilepsy research community is starting to think beyond the traditional approach of using "blunderbuss" seizure-suppressing drugs (e.g., sodium channel blockers) and look at other compounds (both novel and re-purposed chemical entities) that may tackle the fundamental molecular defect of an individual's epilepsy [9]. 
The most recent example of this new approach to epilepsy therapeutics is the approval of the mTOR (mammalian/mechanistic target of rapamycin) inhibitor everolimus as a treatment for epilepsy associated with tuberous sclerosis complex (TSC). The mTOR pathway is a key homeostatic regulatory pathway involved in cell growth and cell replication. TSC is an autosomal dominant multisystem neurocutaneous disorder characterised by hamartomas in multiple organs, including the brain and kidney [19]. It has a prevalence of around 1:10,000 individuals. TSC is caused by mutations in genes coding hamartin (on chromosome 9) and tuberin (on chromosome 16), and is an important cause of (usually drug-refractory) epilepsy often but not always associated with intellectual disability and behavioural problems. Both hamartin and tuberin are important proteins that are involved in homeostatically regulating the mTOR signalling pathway, and mutations in these proteins may result in mTOR overactivity and tumour formation. mTOR inhibitors such as everolimus may help to reverse the molecular defect associated with TSC and ameliorate the clinical consequences of the disorder [20]. Everolimus has already been approved for the treatment of subependymal giant cell astrocytomas and renal angiomyolipomas in patients with TSC. Recently, the EXIST-3 trial has shown that everolimus is independently beneficial in treating the epilepsy associated with TSC [21]. In a study involving 366 patients aged between 2 and 65 years with TSC, comparing placebo and two different exposure doses of everolimus, patients on active treatment had a significant reduction in seizure frequency. The $50 \%$ responder rates in this 18 -week trial were $15.1 \%$ in the placebo group, $28.2 \%$ in the low-exposure everolimus group, and $40.0 \%$ in the high-exposure everolimus group [21].

Other more anecdotal evidence for other examples of precision therapeutics in a number of epilepsies have appeared in the literature and at scientific meetings in the last few years, but none have the rigour or patient number equivalent to the EXIST-3 trial of everolimus in TSC. These examples generally consist of case reports or small case series, and examples include the use of quinidine in KCNT1-associated migrating partial seizures of infancy [2224], the use of memantine in patients with NMDA subunit/GRIN2A-associated epilepsy, and the previously mentioned GLUT-1 deficiency [25].

\section{The Genetic Overlap between Rare and Common Epilepsies Provides Hope for Wider Potential of Precision Therapeutics in Epilepsy}

To systematise precision diagnostics in non-cancer human diseases, an overarching priority is developing the frameworks to quantitatively assess the confidence that a specific variant found in an established disease gene actually contributes to disease in the carrier patient. To this effect, epilepsy is an ideal common disease candidate for precision medicine due to its amenability to genetic discovery (precision diagnostics) and accessible in vitro and in vivo functional models that allow for the characterisation of patient variants and subsequent evaluation of resulting therapeutic opportunities. Given the evidence of overlapping molecular basis between rare and common epilepsies, the current epilepsy precision medicine paradigm, which is focused on treatment of severe rare epilepsies, might have wider applications than previously thought.

Large-scale international efforts such as Epi4K, EGI, and more recently EPI25 continue to discover new epilepsy risk genes. While not a primary aim for some of these efforts, they all facilitate an increase in the number of patients that obtain a molecular diagnosis, thus creating a new opportunity to develop an infrastructure that supports formalising clinician-driven patient outcome registries to maintain careful phenotyping and prospectively report treatment outcomes linked to individuals with secure molecular diagnoses. Such standardised registries will empower large-scale studies investigating the extent of genotype-phenotype 
correlations within individual epilepsy genes and the relationships between molecular diagnoses and existing/novel therapeutic compounds [26]. The expert curation of patient-ascertained presumed pathogenic variants will also be of major research value since a more complete knowledge of the full disease-associated allelic series in epilepsy genes will facilitate appropriate research-driven functional studies.

\section{eHealth as a Catalyst for Precision Therapeutics}

For the purpose of this review we define the term "eHealth" as the intersection of medical informatics, public health, and research, referring to health services and information delivered or enhanced through internet-related technologies (definition adapted from [27]). Electronic health records (EHRs) are a classic example of eHealth infrastructure. They are developed using medical informatics processes to complement, or in some cases replace, traditional paper records. They are embedded in public health infrastructure to improve efficiency and effectiveness of patient care. They provide opportunities for research by providing a rich source of clinical information in a readily available format (where data is appropriately managed and consented). EHRs also provide opportunity for business activity, with growing markets for development, management, and enhancement of EHR solutions.

The increasing adoption of EHRs in clinical practice presents an important opportunity for precision medicine specifically. EHRs can allow access to large volumes of patient-level longitudinal data. As precision medicine is heavily based on correlations between biomarker (e.g., genotype) and clinical phenotype, the advantages that EHR infrastructures can present are obvious, in particular when they are overlaid on, or connected to, programmes of biomarker research. The ability to transition improved diagnostic capacity to a change in prognosis is, in part, dependent on correlation of the allelic series with longitudinal clinical outcome data. EHRs can facilitate this process by, for example, linking to and populating patient registries that track outcomes related to specific genes.

The emergence of electronic patient portals and wearable technologies are set to help bridge the gap in EHR data which is typically captured and recorded during discrete or episodic clinical encounters. Patient-reported outcome measures recorded directly to the EHR from the patient's day-to-day life will further enrich EHR-based phenotyping, recognising the challenges around ensuring data quality. In epilepsy, examples include apps for promoting and recording medication compliance and tools for objective reporting of changes in seizure frequency and/or severity in response to therapy.

EHRs can also play an important role in addressing the challenges with interpretation of sequence data and translating information about actionable genetic variation into clinical practice. Identifying the correct variant contributing to disease risk in a patient involves multidisciplinary collaboration between neurologists, clinical geneticists, bioinformaticians, and genetic scientists, all working within the ACMG framework (see above). Candidate pathogenic gene variants are assessed with reference to the individual's phenotype and a variety of public archives of genetic variation to determine the likelihood of an association. As EHRs allow simultaneous access to multiple users from a variety of locations, they provide a streamlined way of presenting the ensemble of phenotype and genotype information to support multidisciplinary interpretation. Where valid actionable genetic findings are identified, making these available in the patient's EHR supports informed and safe clinical decision making. Making such actionable information available when and where needed to authorised clinicians via the EHR advances personalised care and promotes improved patient safety as underlying genetics will be considered in the patient's diagnosis and subsequent treatment planning. 
However, there are simultaneous challenges with EHR infrastructure and research. Patient confidentiality and data rights must be respected and managed appropriately. A lack of interoperability between different EHRs may limit the comprehensiveness of patient data. For example, an individual's health care may cross multiple organisational boundaries and hence involve multiple EHR instances. Furthermore, the validity of the phenotypes may be uncertain if the query used in the EHR-based case ascertainment is not appropriately defined [26]. These challenges are not insurmountable, but must be considered when exploiting EHR technology to enhance genomic medicine.

\section{Examples of eHealth Infrastructure Facilitating Precision Medicine}

At a national level, the US-based Electronic Medical Records and Genomics (eMERGE) Network is perhaps the best example of a consortium working to overlay EHR data with genomic research, including how best to address the challenges outlined above [28]. The consortium uses data warehousing to safely extract and combine data from multiple EHRs and make aggregate totals available to approved researchers. Similarly, their work involves development and validation of algorithms for robust EHR-based phenotyping. The impact of eMERGE is reflected in the more than 500 research publications it has facilitated to date, including multiple significant genomic discoveries [29].

The Global Alliance for Genomics and Health is providing guidance and leadership for the development of harmonised approaches for the safe, effective, and responsible sharing of genomic and clinical data at the international level. Through a number of focused working groups (Clinical, Data, Security, Regulatory, and Ethics), the Alliance is producing research tools of relevance for genomic medicine and precision therapeutics. Examples of tools produced by the Global Alliance for Genomics and Health include the "Beacon Network," an application programming interface which allows the user to query whether participating organisations have observed particular mutations in their datasets [30]. The Global Alliance has also produced a catalogue of global activities in eHealth, with the aim of identifying and aggregating global resources for sharing genomic and clinical eHealth data [31].

\section{eHealth Infrastructure as a Facilitator of Epilepsy-Related Research}

The Epilepsy Phenome-Genome Project (EPGP) is a large multi-institutional, multinational collaborative research project with the aim of identifying novel genes that cause epilepsy. Over a 7-year recruitment period, the consortium recruited over 4,100 people with epilepsy and their family members. EPGP researchers have developed a range of Web-based informatics applications to facilitate collection of clinical data, tracking of participants and specimens, management of data, review and validation of phenotypic data, and storage of electroencephalograph and neuroimaging data [32]. The informatics dataset contains over 38,000 study activities (EEGs, interviews, etc.), more than 6.9 million data points, and has facilitated the discovery of several new epilepsy genes [33].

An example of an epilepsy-related eHealth infrastructure that facilitates both epilepsy research and the clinic is the Irish Epilepsy EHR [34]. This system was designed specifically for epilepsy and is now used across adult epilepsy tertiary referral centres in Ireland. To date, the system houses clinical details on over 8,000 people with epilepsy, many of whom have consented to research studies. The EHR has been developed to facilitate multidisciplinary meetings, presenting genomic and phenotype data and recording conclusions on candidate variants identified. 


\section{Concluding Comments}

Although precision therapeutics is an exciting new landscape in epileptology, many challenges lie ahead. These include physician education on the new genomics, availability, interpretation and financing of next-generation sequencing, in vitro functional testing models of the potential pathogenicity of individual mutations and their pharmacological modulation, and large collaborative register-based efforts to monitor the outcomes of therapeutic trials of precision therapies, including safety monitoring and the avoidance of "fast and loose" therapies that may follow individual false-positive case reports. However, despite the challenges, the road ahead is full of promise to deliver targeted therapies to an increasing number of patients with epilepsy. In addition, these challenges and opportunities of the new era of genomic medicine also apply to other chronic neurological disorders (e.g., multiple sclerosis). There is a need for coordination at national, EU, and international levels, to allow for a policy and funding frameworks to facilitate new precision medicine over the coming years.

\section{Disclosure Statement}

S.P. serves on the advisory board and is an equity holder of Pairnomix. G.L.C., M.F., and N.D. declare no conflicts of interest.

\section{References}

1 DiLuca M, Olesen J: The cost of brain diseases: a burden or a challenge? Neuron 2014;82:1205-1208.

-2 Laxer KD, Trinka E, Hirsch LJ, Cendes F, Langfitt J, Delanty N, Resnick T, Benbadis SR: The consequences of refractory epilepsy and its treatment. Epilepsy Behav 2014;37:59-70.

-3 EpiPM Consortium: A roadmap for precision medicine in the epilepsies. Lancet Neurol 2015;14:1219-1228.

4 Maljevic S, Reid CA, Petrou S: Models for discovery of targeted therapy in genetic epileptic encephalopathies. J Neurochem 2017;143:30-48.

-5 Leenen LA, Wijnen BF, de Kinderen RJ, van Heugten CM, Evers SM, Majoie MH: Are people with epilepsy using eHealth-tools? Epilepsy Behav 2016;64:268-272.

6 Chung WH, Hung SI, Hong HS, Hsih MS, Yang LC, Ho HC, Wu JY, Chen YT: Medical genetics: a marker for StevensJohnson syndrome. Nature 2004;428:486.

-7 McCormack M, Alfirevic A, Bourgeois S, Farrell JJ, Kasperaviciute D, Carrington M, Sills GJ, Marson T, Jia X, de Bakker PI, et al: HLA-A*3101 and carbamazepine-induced hypersensitivity reactions in Europeans. N Engl J Med 2011;364:1134-1143.

-8 International League against Epilepsy Consortium on Complex Epilepsies: Genetic determinants of common epilepsies: a meta-analysis of genome-wide association studies. Lancet Neurol 2014;13:893-903.

$\checkmark 9$ Cavalleri GL, Delanty N: Opportunities and challenges for genome sequencing in the clinic. Adv Protein Chem Struct Biol 2012;89:65-83.

10 Euro E-RESC, Epilepsy Phenome/Genome P, Epi KC: De novo mutations in synaptic transmission genes including DNM1 cause epileptic encephalopathies. Am J Hum Genet 2014;95:360-370.

11 Epi4K Consortium, Epilepsy Phenome/Genome Project: Ultra-rare genetic variation in common epilepsies: a case-control sequencing study. Lancet Neurol 2017;16:135-143.

12 Goldstein DB, Allen A, Keebler J, Margulies EH, Petrou S, Petrovski S, Sunyaev S: Sequencing studies in human genetics: design and interpretation. Nat Rev Genet 2013;14:460-470.

13 Richards CS, Bale S, Bellissimo DB, Das S, Grody WW, Hegde MR, Lyon E, Ward BE: ACMG recommendations for standards for interpretation and reporting of sequence variations: revisions 2007. Genet Med 2008;10: 294-300.

-14 Traynelis J, Silk M, Wang Q, Berkovic SF, Liu L, Ascher DB, Balding DJ, Petrovski S: Optimizing genomic medicine in epilepsy through a gene-customized approach to missense variant interpretation. Genome Res 2017, Epub ahead of print.

15 Landrum MJ, Lee JM, Benson M, Brown G, Chao C, Chitipiralla S, Gu B, Hart J, Hoffman D, Hoover J, et al: ClinVar: public archive of interpretations of clinically relevant variants. Nucleic Acids Res 2016;44:D862-D868.

$\checkmark 16$ McTague A, Howell KB, Cross JH, Kurian MA, Scheffer IE: The genetic landscape of the epileptic encephalopathies of infancy and childhood. Lancet Neurol 2016;15:304-316. 
-17 Xu X, Yang X, Wu Q, Liu A, Yang X, Ye AY, Huang AY, Li J, Wang M, Yu Z, et al: Amplicon resequencing identified parental mosaicism for approximately $10 \%$ of "de novo" SCN1A mutations in children with Dravet syndrome. Hum Mutat 2015;36:861-872.

18 Klepper J, Scheffer H, Leiendecker B, Gertsen E, Binder S, Leferink M, Hertzberg C, Nake A, Voit T, Willemsen MA: Seizure control and acceptance of the ketogenic diet in GLUT1 deficiency syndrome: a 2- to 5-year followup of 15 children enrolled prospectively. Neuropediatrics 2005;36:302-308.

19 Caban C, Khan N, Hasbani DM, Crino PB: Genetics of tuberous sclerosis complex: implications for clinical practice. Appl Clin Genet 2017;10:1-8.

20 Citraro R, Leo A, Constanti A, Russo E, De Sarro G: mTOR pathway inhibition as a new therapeutic strategy in epilepsy and epileptogenesis. Pharmacol Res 2016;107:333-343.

-21 French JA, Lawson JA, Yapici Z, Ikeda H, Polster T, Nabbout R, Curatolo P, de Vries PJ, Dlugos DJ, Berkowitz N, et al: Adjunctive everolimus therapy for treatment-resistant focal-onset seizures associated with tuberous sclerosis (EXIST-3): a phase 3, randomised, double-blind, placebo-controlled study. Lancet 2016;388:21532163.

22 Bearden D, Strong A, Ehnot J, DiGiovine M, Dlugos D, Goldberg EM: Targeted treatment of migrating partial seizures of infancy with quinidine. Ann Neurol 2014;76:457-461.

23 Chong PF, Nakamura R, Saitsu H, Matsumoto N, Kira R: Ineffective quinidine therapy in early onset epileptic encephalopathy with KCNT1 mutation. Ann Neurol 2016;79:502-503.

24 Bearden D, DiGiovine M, Dlugos D, Goldberg E: Reply. Ann Neurol 2016;79:503-504.

25 Striano P, Vari MS, Mazzocchetti C, Verrotti A, Zara F: Management of genetic epilepsies: from empirical treatment to precision medicine. Pharmacol Res 2016;107:426-429.

26 Smoller JW: The use of electronic health records for psychiatric phenotyping and genomics. Am J Med Genet B Neuropsychiatr Genet 2017, Epub ahead of print.

27 Eysenbach G: What is e-health? J Med Internet Res 2001;3:E20.

28 Smoller JW, Karlson EW, Green RC, Kathiresan S, MacArthur DG, Talkowski ME, Murphy SN, Weiss ST: An eMERGE Clinical Center at Partners Personalized Medicine. J Personalized Medicine 2016;6:E5.

29 eMERGE. https://emerge.mc.vanderbilt.edu/.

30 The Beacon Network. 2017.

31 The Global Alliance for Genomics and Health. 2017.

-32 Nesbitt G, McKenna K, Mays V, Carpenter A, Miller K, Williams M; EPGP Investigators: The Epilepsy Phenome/ Genome Project (EPGP) informatics platform. Int J Med Inform 2013;82:248-259.

33 Project TEPG: EPGP. 2017.

-34 Fitzsimons M, Dunleavy B, O’Byrne P, Dunne M, Grimson J, Kalra D, Normand C, Delanty N: Assessing the quality of epilepsy care with an electronic patient record. Seizure 2013;22:604-610. 